\title{
Negative correlation between retinal displacement and ganglion cell layer thickness changes in eyes with epiretinal membrane
}

European Journal of Ophthalmology $1-8$

(C) The Author(s) 2019 Article reuse guidelines: sagepub.com/journals-permissions DOI: $10.1177 / 1 \mid 20672119894887$ journals.sagepub.com/home/ejo (S)AGE

\author{
Pasquale Loiudice' (D), Marco Pellegrini², \\ Andrea Montesel' ${ }^{\text {DD }}$, Marco Nardi ', Enrico Peiretti ${ }^{3}$, \\ Stefano De Cillà ${ }^{4}$, Chiara Posarelli', Michele Figus' ${ }^{\text {(iD }}$ \\ and Giamberto Casini ${ }^{1}$
}

\begin{abstract}
Purpose: To examine retinal displacement after vitrectomy with internal limiting membrane peeling for epiretinal membrane treatment, and to determine the correlation between the extent of displacement and the ganglion cell layer thickness changes.

Methods: Thirty-nine consecutive patients were evaluated. Optical coherence tomography was performed preoperatively, and 2 and 6 months postoperatively. The distance between the fovea, the vessel intersection in the superior nasal, superior temporal, inferior nasal, and inferior temporal macular regions, and the optic disk were measured. Ganglion cell layer thickness was recorded.

Results: The distance from the optic disk to the fovea and the superior nasal and inferior nasal vessel intersections significantly reduced at 2 and 6 months. Ganglion cell layer thickness significantly decreased in all the subfields (except nasal) at 6 months. A significant negative correlation was found between the change in the ganglion cell layer thickness and the movement of the fovea toward the optic disk. The ganglion cell layer thickness changes significantly correlated with retinal displacement.

Conclusion: Retinal displacement toward the optic disk was detected after vitrectomy with internal limiting membrane peeling in eyes with epiretinal membrane. Ganglion cell layer thickness significantly reduced in all the subfields (except nasal). The extent of the displacement and the ganglion cell layer thickness changes showed significant negative correlation.
\end{abstract}

\section{Keywords}

Epiretinal membrane, ganglion cell layer, internal limiting membrane peeling, retinal displacement

Date received: 25 May 2019; accepted: 24 November 2019

\section{Introduction}

Idiopathic epiretinal membrane (ERM) is a fibrotic process on the surface of the retina, due to fibrocellular proliferation at the vitreoretinal interface, ranging from a thin translucent layer to a thick opaque membrane. ${ }^{1,2}$ Transdifferentiation of cellular components into myofibroblasts is responsible for the contractile properties of the membrane. ${ }^{3,4}$ As the disease progresses, it causes wrinkling and distortion of the retinal surface and vascular tortuosity. At this stage, ERM is also called macular pucker.

Several authors have described retinal movement due to the contraction of the ERM. Weinberger et al. ${ }^{5}$ used digital
'Ophthalmology Unit, Department of Surgical, Medical, Molecular Pathology and of Critical Area, University of Pisa, Pisa, Italy 2Ophthalmology Unit, Department of Experimental, Diagnostic and Specialty Medicine, S.Orsola-Malpighi Teaching Hospital, University of Bologna, Bologna, Italy

${ }^{3}$ Department of Surgical Sciences, Eye Clinic, University of Cagliari, Cagliari, Italy

${ }^{4}$ Department of Health Sciences, Eye Clinic, University of Piemonte

Orientale, Novara, Italy

Corresponding author:

Michele Figus, Ophthalmology Unit, Department of Surgical, Medical,

Molecular Pathology and of Critical Area, University of Pisa, Via

Paradisa 2, 56/24 Pisa, Pisa, Italy.

Email: figusmichele@gmail.com 
red-free photography to evaluate the displacement of retinal blood vessels during the natural course of ERM formation. Lo et al. ${ }^{6}$ reported foveal dystopia in patients with ERM before surgery and foveal movement toward its expected location postoperatively. Retinal displacement has also been reported after vitrectomy in eyes with macular holes ${ }^{7,8}$ and after retinal detachment surgery. ${ }^{9}$

Patients with ERM are currently treated with pars plana vitrectomy (PPV) and removal of the membrane. ${ }^{10}$ Dyeassisted internal limiting membrane (ILM) peeling can reduce the rate of ERM recurrence. ILM peeling ensures complete removal of the fibrocellular membrane and eliminates the scaffold to prevent further proliferation. ${ }^{10-12}$ However, PPV and ILM peeling may influence inner retinal structure, as a reduction in macular ganglion cell-plexiform layer thickness has been reported after vitrectomy for a macular hole, particularly in patients who underwent ILM peeling. ${ }^{13}$

The purpose of this study was to evaluate retinal movements after Brilliant Blue G-assisted (Brilliant Peel ${ }^{\circledR}$, Fluoron GmbH, Ulm, Germany) ILM peeling for ERM and to determine whether there was a correlation between the degree of displacement and ganglion cell layer (GCL) thickness changes.

\section{Methods}

In this prospective, interventional case series study, we enrolled 39 eyes of 39 consecutive patients diagnosed with ERM and scheduled for surgery. The study received approval from the local Institutional Review Board (Comitato Etico di Area Vasta Nord Ovest, register number: 15255_CASINI), and was conducted in adherence to the tenets of the Declaration of Helsinki. Every patient signed an informed consent form. Eyes with diabetic retinopathy, macular degeneration, myopic chorioretinal atrophy, rhegmatogenous retinal detachment, and any other disease that potentially could interfere with measurements were excluded.

All patients underwent comprehensive ophthalmologic examinations, including measurement of best-corrected visual acuity (BCVA), using the Early Treatment Diabetic Retinopathy Study (ETDRS) visual acuity chart, intraocular pressure, and slit-lamp biomicroscopy of the anterior and posterior segments. All surgeries were performed by a single surgeon (G.C.) using the Alcon Constellation Ultrahigh-Speed Vitrectomy 25-gauge total pack (Alcon, Fort Worth, TX, United States). A BIOM viewing system (Oculus, Wetzlar, Germany) or a Resight 700 (Carl Zeiss Meditec AG, Jena, Germany) was used for posterior visualization. The surgical treatment included 25-gauge PPV, posterior vitreous detachment, peeling of the ERM, dyeassisted peeling of the ILM using Brilliant Blue G, and air-fluid exchange. The stained ILM was peeled to the edge of the vascular arcade using an end-gripping forceps
(Grieshaber Asymmetrical Forceps, DSP, Alcon, Fort Worth, TX, United States). All patients remained in prone position for $48 \mathrm{~h}$ after surgery. Phakic patients underwent cataract surgery at the same time as the vitrectomy.

Spectral-domain optical coherence tomography (SD-OCT, Spectralis HRA + OCT; Heidelberg Engineering, Heidelberg, Germany) was performed preoperatively, and 2 and 6 months after surgery. Infrared images were used to calculate the relative positions of optic disk, fovea, and retinal vessels. Using the caliper function, we measured the distance between the point at which the central retinal vein emerges from the optic disk and the center of the fovea, and the distance between the optic disk and vessel intersection in the superior nasal, superior temporal, inferior nasal, and inferior temporal macular regions (Figure 1).

The GCL thickness was calculated using the segmentation tool of the instrument. All the segmentations were carefully checked and manually adjusted when needed. Unreliable images were excluded from the analysis. The macular area was divided in nine subfields according to the ETDRS grid: foveal, inner superior, inner inferior, inner temporal, inner nasal, outer superior, outer inferior, outer temporal, and outer nasal (Figure 2).

Statistical analysis was performed using IBM SPSS software (IBM, NY, United States). The distribution of data was assessed using Kolmogorov-Smirnov test and Shapiro-Wilk test. The Wilcoxon test was used to determine whether differences over time were significant. The correlations between the degree of displacement and GCL thickness changes were evaluated using Spearman rank correlations. Differences were considered significant when $P<0.05$.

\section{Results}

In this prospective observational study, 39 eyes of 39 patients (21 male and 18 female) were consecutively enrolled. Mean age was $69.35 \pm 0.7$ years. Of the 39 patients, $15(38.46 \%)$ were phakic and underwent combined phacovitrectomy.

Table 1 shows the median of the distances from the optic disk to the fovea, and to the superior nasal, superior temporal, inferior nasal, and inferior temporal vessel intersections before surgery, and 2 and 6 months after surgery. Two months after surgery, the median of the distance from the optic disk to the fovea was reduced by $100 \mu \mathrm{m}(-3 \%)$ compared with preoperative values, and the median of the distances between the fovea and the superior nasal, inferior nasal, superior temporal, and inferior temporal vessel intersections were increased by $94 \mu \mathrm{m}(4 \%), 117 \mu \mathrm{m}(7 \%)$, and $193 \mu \mathrm{m}(9 \%)$, compared with preoperative values. The reduction in distance between the optic disk and the fovea and between the optic disk and the superior nasal and inferior nasal vessel intersections were statistically significant ( $P=0.008, P=0.037$, and $P=0.005$, respectively, Wilcoxon 


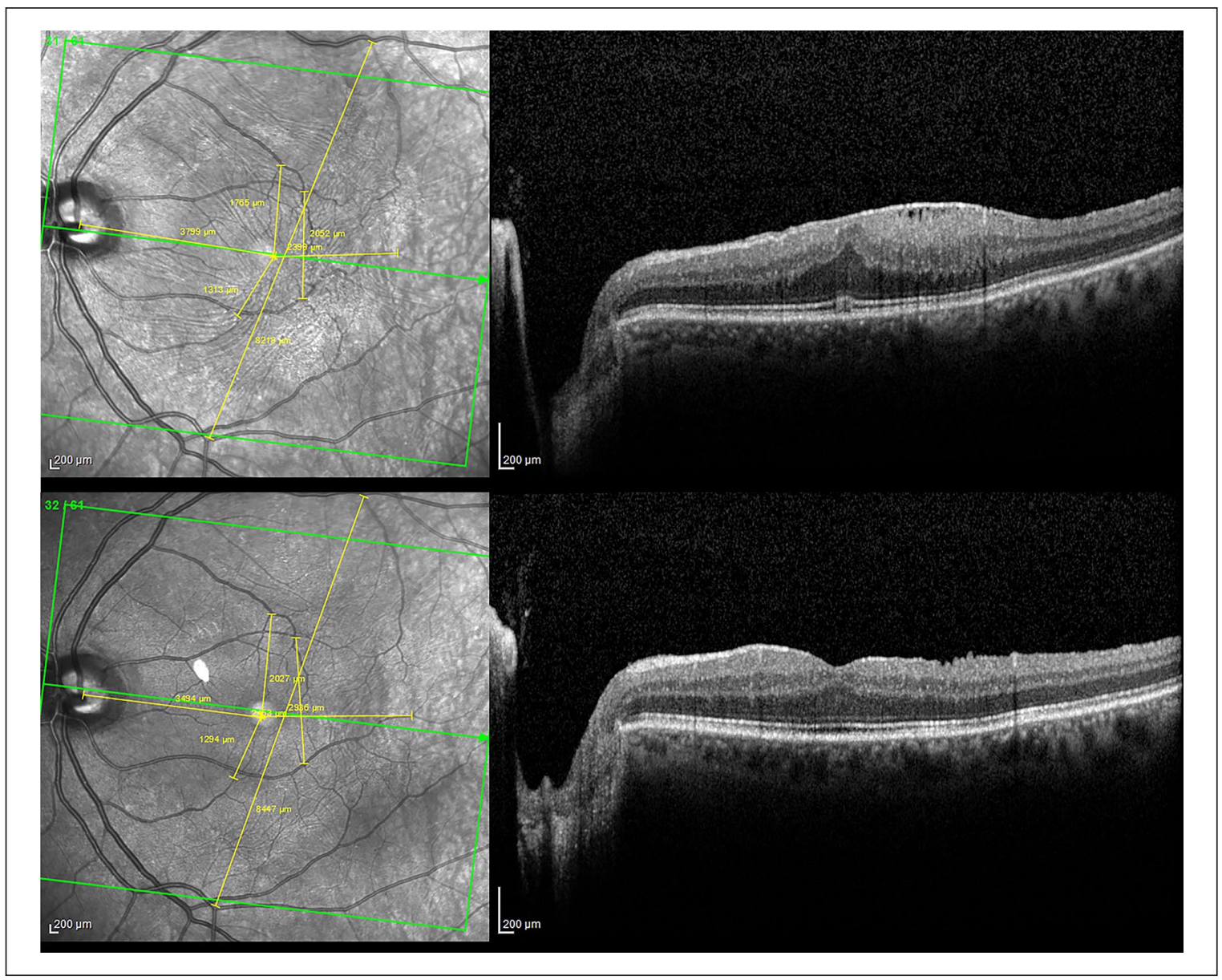

Figure I. Fundus infrared images and spectral-domain optical coherence tomographic images in a patient with idiopathic epiretinal membrane. The distance from the optic disk to the fovea and from the optic disk to the vessel intersection in the superior nasal, inferior nasal, superior temporal, and inferior temporal macular regions were manually measured, preoperatively (upper left) and 6 months after surgery (lower left).

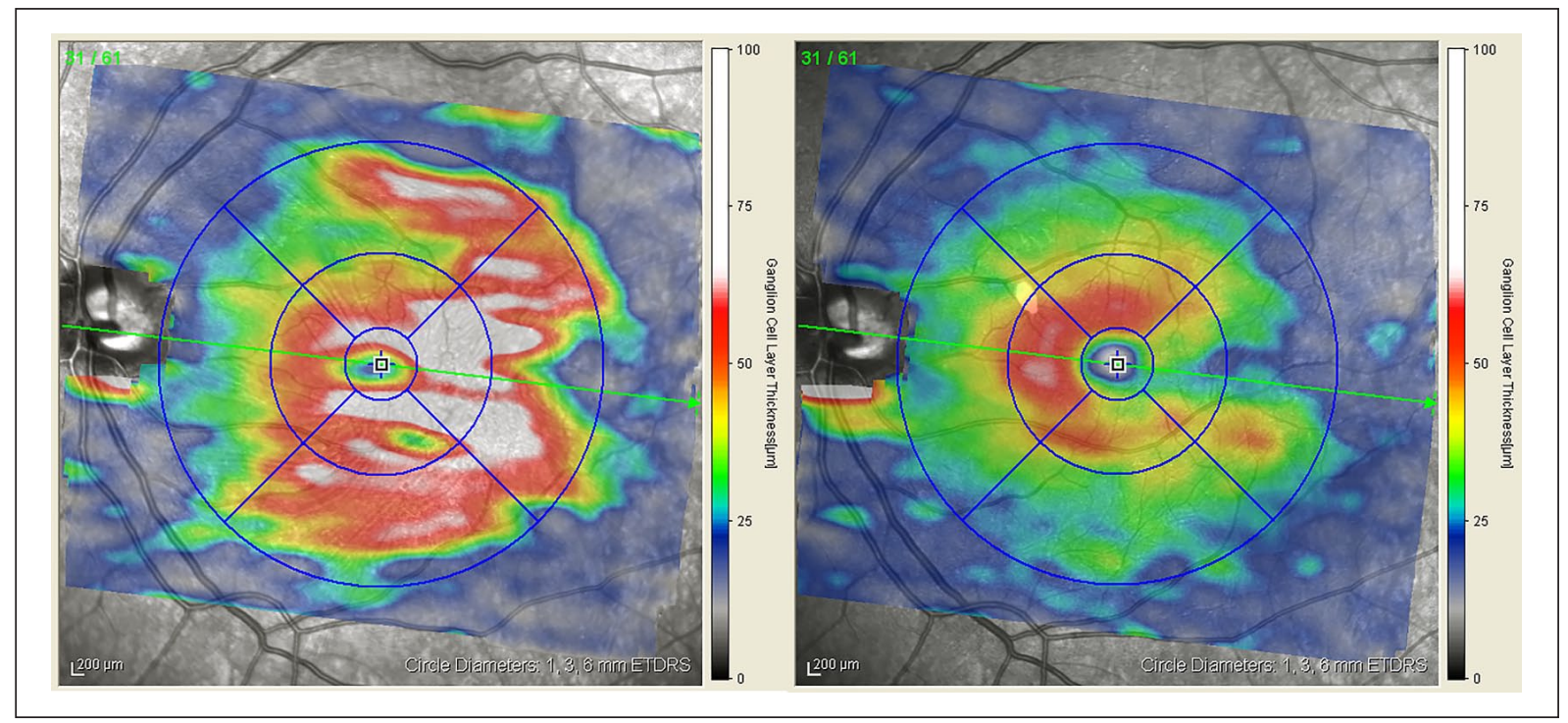

Figure 2. Ganglion cell layer thickness preoperatively (left) and 6 months (right) after vitrectomy with inner limiting membrane peeling for epiretinal membrane. The macular region was divided in nine subfields according to Early Treatment Diabetic Retinopathy Study grid. 
Table I. Retinal measurements.

\begin{tabular}{|c|c|c|c|c|c|}
\hline Distance & TO & TI & $\mathrm{Pa}$ & $\mathrm{T} 2$ & $P^{b}$ \\
\hline F-OD & 3485 (2989-4342) & 3384 (2932-3970) & 0.002 & $3257(2895-3946)$ & 0.001 \\
\hline F-SUP & $2156(1442-2850)$ & 2250 (I499-3470) & 0.049 & $2375(1462-3824)$ & 0.035 \\
\hline F-INF & 1648 (836-2777) & 1765 (1019-2829) & 0.046 & 1879 (1017-2929) & 0.025 \\
\hline F-TEMP & $3084(194 I-3668)$ & 3277 (I972-393I) & 0.018 & $3419(1891-4167)$ & 0.003 \\
\hline MIN & $3117(1883-4440)$ & $3189(2234-5217)$ & 0.003 & 3206 (2342-5339) & 0.004 \\
\hline $\mathrm{MAJ}$ & $7595(5|53-86| 5)$ & 7778 (5292-858I) & 0.026 & 7899 (5394-8573) & 0.016 \\
\hline
\end{tabular}

Distances $(\mu \mathrm{m})$ from the fovea $(F)$ to the optic disk (OD) and from the $F$ to the vessel intersection in the superior (SUP), inferior (INF), temporal (TEMP) macular region, distance between minor vessel branches (MIN) and major vessel branches (MAJ) at baseline (T0), 2 months (TI) and 6 months (T2) after surgery. Values are median (minimum-maximum).

a2 months after surgery compared to baseline with Wilcoxon test.

b6 months after surgery compared to baseline with Wilcoxon test.

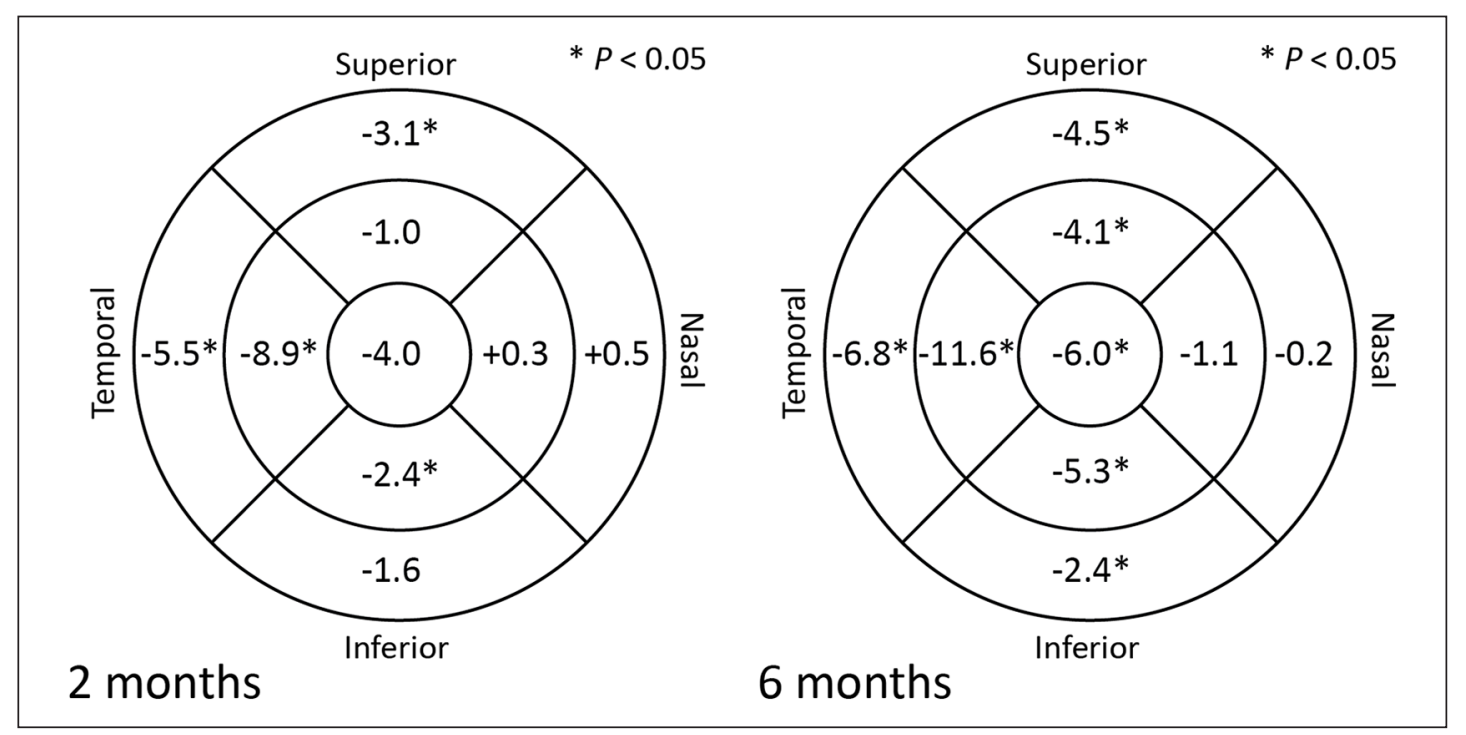

Figure 3. Mean changes in the ganglion cell layer thickness at 2 and 6 months after epiretinal membrane surgery compared with preoperative values. Asterisks indicate $\mathrm{P}<0.05$ (Wilcoxon tests).

$\mathrm{T} 0=$ baseline; $\mathrm{TI}=2$ months postoperatively; $\mathrm{T} 2=6$ months postoperatively.

test). Six months after surgery, the mean distance between the optic disk and the fovea was reduced by $166 \mu \mathrm{m}(4.8 \%)$ compared with preoperative values, and the distance from the optic disk to the superior nasal, inferior nasal, superior temporal, and inferior temporal vessel intersections was reduced by $62 \mu \mathrm{m}(2.2 \%), 75 \mu \mathrm{m}(2.8 \%), 35 \mu \mathrm{m}(1.5 \%)$, and $18 \mu \mathrm{m}(0.3 \%)$, respectively, compared with preoperative values. The reduction in distance from the optic disk to the fovea and from the optic disk to the superior nasal and inferior nasal vessel intersections were statistically significant $(P=0.006, P=0.043$, and $P=0.039$, respectively, Wilcoxon test).

Table 2 shows the mean GCL thickness in nine macular subfields before surgery, and 2 and 6 months after surgery. The mean changes in the GCL thickness 2 and 6 months after surgery compared with preoperative values are shown in Figure 3. Two months after surgery, the GCL thickness significantly decreased in the outer temporal, inner temporal, outer superior, and inner inferior macular subfields compared with preoperative values $(P<0.001$, $P<0.001, P=0.043$, and $P=0.039$, respectively, Wilcoxon test). Six months after surgery, the GCL thickness significantly decreased in the fovea and in the outer temporal, inner temporal, outer superior, inner superior, outer inferior, and inner inferior macular subfields compared with preoperative values $(P=0.004, P<0.001, P<0.001$, $P=0.001, P=0.024, P=0.039$, and $P=0.004$, respectively, Wilcoxon test).

Postoperative GCL thinning was notably higher in the temporal section. Before surgery, the mean nasal GCL thickness was $40.9 \pm 6.8 \mu \mathrm{m}$ and the mean temporal GCL thickness was $41.3 \pm 8.1 \mu \mathrm{m}$, which was not a statistically significant difference ( $P=0.797$, Wilcoxon test). However, 2 months after surgery, a statistically significant difference of $6.7 \pm 8.4 \mu \mathrm{m}$ was found between the mean nasal and the mean temporal GCL thickness $(P<0.001$, Wilcoxon test). 
Table 2. Ganglion cell layer measurements.

\begin{tabular}{|c|c|c|c|c|c|}
\hline & TO & TI & $\mathrm{Pa}$ & $\mathrm{T} 2$ & $p^{b}$ \\
\hline Central & $32(9-65)$ & $30(13-60)$ & 0.091 & 28.5 (13-59) & 0.004 \\
\hline Outer nasal & $32.5(20-40)$ & $32(24-4 I)$ & 0.507 & $32(22-46)$ & 0.686 \\
\hline Inner nasal & $47.5(26-68)$ & $49(27-67)$ & 0.957 & $48(25-73)$ & 0.808 \\
\hline Outer temporal & $30(18-62)$ & $28(17-54)$ & $<0.001$ & $27(17-47)$ & $<0.001$ \\
\hline Inner temporal & $44.5(27-67)$ & $36.5(16-57)$ & $<0.001$ & $35.5(16-58)$ & $<0.001$ \\
\hline Outer superior & $30(24-54)$ & $28.5(20-44)$ & 0.043 & $28(20-37)$ & 0.001 \\
\hline Inner superior & $49.5(23-67)$ & $45.5(23-66)$ & 0.588 & $43(23-66)$ & 0.024 \\
\hline Outer inferior & $28.5(21-48)$ & $27(2 \mathrm{I}-32)$ & 0.560 & $26.5(11-34)$ & 0.039 \\
\hline Inner inferior & $48(10-65)$ & $46(27-62)$ & 0.039 & $42(25-60)$ & 0.004 \\
\hline
\end{tabular}

Ganglion cell layer thickness $(\mu \mathrm{m})$ in the foveal, outer nasal, inner nasal, outer temporal, inner temporal, outer superior, inner superior, outer inferior, and inner inferior macular area before surgery (T0), 2 months (TI), and 6 months (T2) postoperatively. Values are median (minimum-maximum). a 2 months after surgery compared to baseline with Wilcoxon test.

b6 months after surgery compared to baseline with Wilcoxon test.

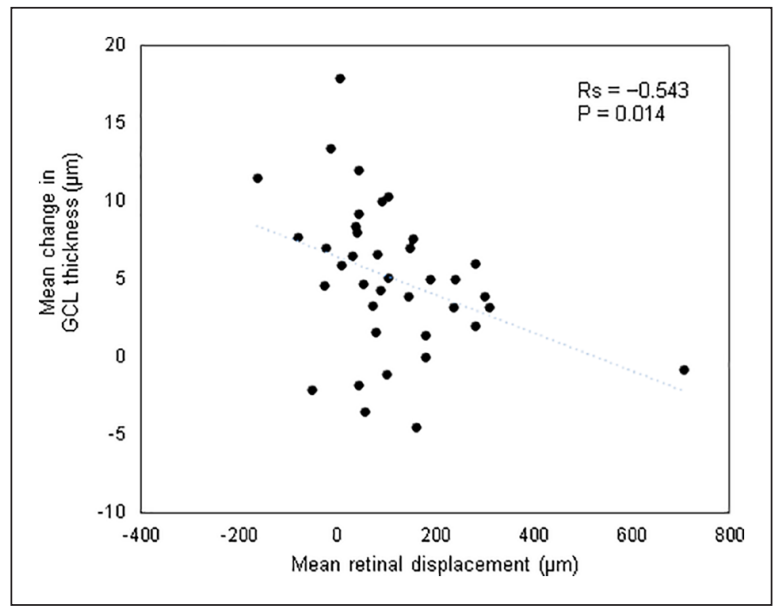

Figure 4. Scatter plot graph showing negative correlation between mean change in ganglion cell layer thickness and mean retinal displacement.

This difference increased to $7.7 \pm 8.2 \mu \mathrm{m}$ at 6 months after surgery $(P=0.001$, Wilcoxon test $)$.

In the postoperative period, we observed two cases of macular edema. Central macular thickness was 280 and $341 \mu \mathrm{m}$, respectively. The patients were treated with intravitreal injection of triamcinolone acetonide $\left(\right.$ Taioftal ${ }^{\mathbb{R}}$, SOOFT S.p.A., Italy). The edema was completely resolved at the last follow-up. No other complications occurred.

A Spearman correlation was performed to determine the relationship between the movement of the retina toward the optic disk and the change in the GCL thickness. A significant negative correlation was found between the change in the GCL thickness in the inner temporal area and the movement of the fovea toward the optic disk ( $P=0.024$; $R=-0.379$ ). Moreover, the mean of the GCL thickness changes in all the subfields was significantly correlated with the mean of the retinal displacement in all the measured areas ( $P=0.014 ; R=-0.543)$ (Figure 4).

\section{Discussion}

Patients with ERM experience progressive worsening of visual function due to the thickening and contraction of the membrane and the distortion of the underlying retina. These modifications may result in metamorphopsia and loss of central vision. Arimura et al. ${ }^{14}$ found a correlation between the severity of metamorphopsia and retinal contraction in eyes with ERM. In cases of ERM, the contraction of the membrane dislocates the photoreceptors and the outer retinal layer, with consequent metamorphopsia that can still be present even after surgically successful ERM peeling and visual acuity improvement. ${ }^{15}$

Topographic modifications of retinal vessels have previously been observed in eyes with ERM.5,16,17 In our study, we observed that the retina in the macular area moved toward the optic disk after PPV and ILM peeling. We found a significant reduction in distance between the optic disk and the fovea, and between the optic disk and the superior nasal and inferior nasal vessels. No significant change was observed in the temporal area.

This is not completely in accordance with previous studies. Retinal displacement toward the optic disk was previously reported after PPV with ILM peeling for macular hole $^{7}$; however, Ishida et al. ${ }^{8}$ reported that the temporal vessels moved more than the nasal ones. In a comparative study of retinal displacement after surgery for macular hole and ERM, Rodrigues et al..$^{18}$ found relative retinal expansion after ERM removal and contraction after macular hole closure, due to, respectively, centrifugal and centripetal forces acting on the retina after surgery. Using red-free digital photography, Weinberger et al. ${ }^{5}$ studied the displacement of retinal blood vessels during the natural course of ERM formation. They found that the distances between vascular arcades may either increase or decrease or show a mixed pattern. To avoid this possible bias, in our study, we use the fovea as a landmark, and we measured the relative distance between the fovea and the optic disk and the 
vascular arcade. In this way, we were able to independently measure the re-arrangement forces according with the direction in which they were acting. Centrifugal movements have been observed in eyes with ERM due to multiple foci of retinal traction. ${ }^{17}$

A possible explanation for similar behavior may be found if we consider the optic disk as a fulcrum or an anchor point for the retina. Under the action of the ERM, the retinal surface contracts and distorts. The removal of the ERM modifies the tangential stress forces, so the retina may relax and recover to its original arrangement. ${ }^{19}$ Since the optic disk is a fixed point, the retina behaves like a spring and displacement movements are more evident in foveal and nasal regions. This also explains why retinal dislocation was not significant in the temporal area. Kawano et al. ${ }^{7}$ hypothesized that, in cases of macular hole, ILM peeling was likely to cause retinal movement. They suggested that the ILM peeling may alter the forces acting on the retina, and since there is no ILM on the optic disk, the fovea was displaced nasally.

Progress in SD-OCT technology has made it possible to obtain high-resolution retinal images; furthermore, the development of a segmentation tool in the software enables a layer-by-layer analysis. ${ }^{20,21} \mathrm{~A}$ high reproducibility of the measurements of the retinal ganglion cell-inner plexiform layer using SD-OCT in both normal and glaucomatous eyes has been previously reported.22-24 Auto-segmentation has also shown high repeatability in eyes with ERM and central macular thickness less than $450 \mu \mathrm{m} .{ }^{25}$

Examination of each retinal layer has increasingly gained interest since a correlation between inner retinal layer and retinal sensibility was reported. ${ }^{26}$ Postoperative visual acuity has been reported to be related to the thickness of the inner retinal layer and changes in the GCL. ${ }^{27,28}$ Baba et al. ${ }^{26}$ observed a reduction in the thickness of the ganglion cell complex after vitrectomy with ILM peeling in eyes with idiopathic macular hole (IMH).

Peeling the ILM is an effective and widely performed procedure that offers numerous advantages. It ensures complete removal of the ERM and is associated with an increase in closure rate in macular hole surgery, by removing a potential scaffold for cell migration. ${ }^{29}$ However, several authors have reported that the retina may be affected by ILM peeling. ILM peeling has been reported to result in defects of the inner retina, as evidenced by an increase in the dissociated optic nerve fiber layer appearance. ${ }^{30-32}$ Decreased retinal sensitivity, ${ }^{33}$ microscotomas, ${ }^{33}$ swelling of the arcuate retinal nerve fiber layer, ${ }^{34}$ and reduction of the thickness of the ganglion cell complex ${ }^{26}$ have also been associated with ILM peeling.

In our study, the GCL thickness significantly reduced in the outer temporal, inner temporal, outer superior, and inner inferior subfields at 2 months postoperatively and in all sectors except the nasal ones at the last follow-up. This indicates that retinal modifications continued up to 6 months after surgery.
Postoperative GCL thinning was notably higher in the temporal section and the difference in thickness between the mean temporal and the nasal GCL increased at 2 months and further decreased at 6 months postoperatively, despite no statistically significant difference at baseline. Sevim and Sanisoglu ${ }^{35}$ reported no differences in the thickness of the GCL in eyes with IMH after Brilliant Blue G-assisted ILM peeling. In contrast, Baba et al. ${ }^{26}$ reported a reduction in thickness of the ganglion cell complex after vitrectomy and ILM peeling in patients with IMH.

In their retrospective study, Won et al. ${ }^{36}$ reported a reduction in GCL thickness in the foveal, inner superior, inner temporal, and outer nasal subfields after vitrectomy and ILM peeling for ERM. Lim et al. ${ }^{37}$ studied the effect of vitrectomy in eyes without vitreoretinal disease and found that the thickness of the macula and ganglion cell-inner plexiform layer increased at 1 month postoperatively, after which it returned to preoperative values. These findings are thought to be due to vitreoretinal inflammation caused by the surgery.

The pathogenesis of the GCL modifications has not been fully explained and is currently debated. Mechanical damage induced by forceps during ILM peeling, inflammatory distress, and trophic action carried out by ILM toward the ganglion cells have been hypothesized to have a role in GCL modifications after surgery. ${ }^{36,37}$ Another explanation would be a loss of traumatized Müller cells contained in the GCL, as the endfeet of Müller cells are removed with the ILM peeling. ${ }^{38,39}$

However, GCL changes could be affected by retinal movements that occur after surgery. In our study, we found a significant correlation between retinal displacement and GCL thickness. In other words, the correlation between the mean retinal displacement and the mean GCL thickness change was significant and negative, which indicates that the greater the displacement, the less the GCL thickness decrease. A significant negative correlation was also found between the GCL thickness change in the inner temporal area (the area in which the greatest reduction occurred) and foveal displacement, which was the greater displacement. To the best of our knowledge, no previous studies have analyzed a similar correlation. A possible explanation for this behavior was discussed as follows. It is believed that retinal movements occur at the interface between the retinal pigment epithelium (RPE) and retina, as demonstrated by hyperautofluorescent lines running parallel to major blood vessels, visible on fundus autofluorescence imaging. We observed that in some patients, the retina became less mobile, probably due to a greater adhesion to the RPE. In these cases, the tension forces that drive retinal arrangement after surgery resulted in stretching of the inner retina with resulting decrease in GCL thickness. In eyes with a more mobile retina and a greater displacement after ILM peeling, the stress forces were lower, with consequent lower impact on the GCL. 
In conclusion, we found retinal displacement toward the optic disk after vitrectomy with Brilliant Blue G-assisted ILM peeling in eyes with ERM. The GCL thickness significantly reduced in all the ETDRS subfields except the nasal ones, with the greatest reduction in the temporal area. There was a significant negative correlation between the extent of the displacement and the GCL thickness changes.

\section{Declaration of conflicting interests}

The author(s) declared no potential conflicts of interest with respect to the research, authorship, and/or publication of this article.

\section{Funding}

The author(s) received no financial support for the research, authorship, and/or publication of this article.

\section{ORCID iDs}

Pasquale Loiudice (iD https://orcid.org/0000-0001-8529-9897

Andrea Montesel (iD https://orcid.org/0000-0002-1910-9976

Michele Figus (iD https://orcid.org/0000-0003-2243-9033

\section{References}

1. Smiddy WE, Maguire AM, Green WR, et al. Idiopathic epiretinal membranes. Ultrastructural characteristics and clinicopathologic correlation. Ophthalmology 1989; 96: 811-820; discussion 821 .

2. Zhao F, Gandorfer A, Haritoglou C, et al. Epiretinal cell proliferation in macular pucker and vitreomacular traction syndrome: analysis of flat-mounted internal limiting membrane specimens. Retina 2013; 33: 77-88.

3. Parapuram SK, Chang B, Li L, et al. Differential effects of TGFbeta and vitreous on the transformation of retinal pigment epithelial cells. Invest Ophthalmol Vis Sci 2009; 50: 5965-5974.

4. Guidry C, Bradley KM and King JL. Tractional force generation by human Muller cells: growth factor responsiveness and integrin receptor involvement. Invest Ophthalmol Vis Sci 2003; 44: 1355-1363.

5. Weinberger D, Stiebel-Kalish H, Priel E, et al. Digital redfree photography for the evaluation of retinal blood vessel displacement in epiretinal membrane. Ophthalmology 1999; 106: 1380-1383.

6. Lo D, Heussen F, Ho HK, et al. Structural and functional implications of severe foveal dystopia in epiretinal membranes. Retina 2012; 32: 340-348.

7. Kawano K, Ito Y, Kondo M, et al. Displacement of foveal area toward optic disc after macular hole surgery with internal limiting membrane peeling. Eye 2013; 27: 871-877.

8. Ishida M, Ichikawa Y, Higashida R, et al. Retinal displacement toward optic disc after internal limiting membrane peeling for idiopathic macular hole. Am J Ophthalmol 2014; 157: 971-977.

9. Shiragami C, Shiraga F, Yamaji H, et al. Unintentional displacement of the retina after standard vitrectomy for rhegmatogenous retinal detachment. Ophthalmology 2010; 117: 86-92.e1.
10. Pournaras CJ, Emarah A and Petropoulos IK. Idiopathic macular epiretinal membrane surgery and ILM peeling: anatomical and functional outcomes. Semin Ophthalmol 2011; 26: $42-46$.

11. Jung JJ, Hoang QV, Ridley-Lane ML, et al. Long-term retrospective analysis of visual acuity and optical coherence topographic changes after single versus double peeling during vitrectomy for macular epiretinal membranes. Retina 2016; 36: 2101-2109.

12. Schechet SA, DeVience E and Thompson JT. The effect of internal limiting membrane peeling on idiopathic epiretinal membrane surgery, with a review of the literature. Retina 2017; 37: 873-880.

13. Seo KH, Yu SY and Kwak HW. Topographic changes in macular ganglion cell-inner plexiform layer thickness after vitrectomy with indocyanine green-guided internal limiting membrane peeling for idiopathic macular hole. Retina 2015; 35: $1828-1835$.

14. Arimura E, Matsumoto C, Okuyama S, et al. Retinal contraction and metamorphopsia scores in eyes with idiopathic epiretinal membrane. Invest Ophthalmol Vis Sci 2005; 46: 2961-2966.

15. Bouwens MD and Van Meurs JC. Sine Amsler Charts: a new method for the follow-up of metamorphopsia in patients undergoing macular pucker surgery. Graefes Arch Clin Exp Ophthalmol 2003; 241: 89-93.

16. Yang HK, Kim SJ, Jung YS, et al. Improvement of horizontal macular contraction after surgical removal of epiretinal membranes. Eye 2011; 25: 754-761.

17. Kofod M and la Cour M. Quantification of retinal tangential movement in epiretinal membranes. Ophthalmology 2012; 119: 1886-1891.

18. Rodrigues IA, Lee EJ and Williamson TH. Measurement of retinal displacement and metamorphopsia after epiretinal membrane or macular hole surgery. Retina 2016; 36: 695702.

19. Nitta E, Shiraga F, Shiragami C, et al. Displacement of the retina and its recovery after vitrectomy in idiopathic epiretinal membrane. Am J Ophthalmol 2013; 155: 1014-1020.e1.

20. Mujat M, Chan R, Cense B, et al. Retinal nerve fiber layer thickness map determined from optical coherence tomography images. Opt Express 2005; 13: 9480-9491.

21. Garvin MK, Abramoff MD, Wu X, et al. Automated 3-D intraretinal layer segmentation of macular spectral-domain optical coherence tomography images. IEEE Trans Med Imaging 2009; 28: 1436-1447.

22. Mwanza JC, Oakley JD, Budenz DL, et al. Macular ganglion cell-inner plexiform layer: automated detection and thickness reproducibility with spectral domain-optical coherence tomography in glaucoma. Invest Ophthalmol Vis Sci 2011; 52: 8323-8329.

23. Francoz M, Fenolland JR, Giraud JM, et al. Reproducibility of macular ganglion cell-inner plexiform layer thickness measurement with cirrus HD-OCT in normal, hypertensive and glaucomatous eyes. Br J Ophthalmol 2014; 98 : 322 328.

24. Garas A, Vargha P and Hollo G. Reproducibility of retinal nerve fiber layer and macular thickness measurement with the RTVue-100 optical coherence tomograph. Ophthalmology 2010; 117: 738-746. 
25. Lee HJ, Kim MS, Jo YJ, et al. Thickness of the macula, retinal nerve fiber layer, and ganglion cell layer in the epiretinal membrane: the repeatability study of optical coherence tomography. Invest Ophthalmol Vis Sci 2015; 56: 4554-4559.

26. Baba T, Yamamoto S, Kimoto R, et al. Reduction of thickness of ganglion cell complex after internal limiting membrane peeling during vitrectomy for idiopathic macular hole. Eye 2012; 26: 1173-1180.

27. Koo HC, Rhim WI and Lee EK. Morphologic and functional association of retinal layers beneath the epiretinal membrane with spectral-domain optical coherence tomography in eyes without photoreceptor abnormality. Graefes Arch Clin Exp Ophthalmol 2012; 250: 491-498.

28. Lee EK and Yu HG. Ganglion cell-inner plexiform layer thickness after epiretinal membrane surgery: a spectraldomain optical coherence tomography study. Ophthalmology 2014; 121: 1579-1587.

29. Lois N, Burr J, Norrie J, et al. Internal limiting membrane peeling versus no peeling for idiopathic full-thickness macular hole: a pragmatic randomized controlled trial. Invest Ophthalmol Vis Sci 2011; 52: 1586-1592.

30. Alkabes M, Salinas C, Vitale L, et al. En face optical coherence tomography of inner retinal defects after internal limiting membrane peeling for idiopathic macular hole. Invest Ophthalmol Vis Sci 2011; 52: 8349-8355.

31. Tadayoni R, Paques M, Massin P, et al. Dissociated optic nerve fiber layer appearance of the fundus after idiopathic epiretinal membrane removal. Ophthalmology 2001; 108: 2279-2283.
32. Ito Y, Terasaki H, Takahashi A, et al. Dissociated optic nerve fiber layer appearance after internal limiting membrane peeling for idiopathic macular holes. Ophthalmology 2005; 112: 1415-1420.

33. Tadayoni R, Svorenova I, Erginay A, et al. Decreased retinal sensitivity after internal limiting membrane peeling for macular hole surgery. Br J Ophthalmol 2012; 96: 1513-1516.

34. Clark A, Balducci N, Pichi F, et al. Swelling of the arcuate nerve fiber layer after internal limiting membrane peeling. Retina 2012; 32: 1608-1613.

35. Sevim MS and Sanisoglu H. Analysis of retinal ganglion cell complex thickness after Brilliant Blue-assisted vitrectomy for idiopathic macular holes. Curr Eye Res 2013; 38: $180-184$.

36. Won JY, Kim M and Park YH. Postoperative changes in the retinal thickness and volume after vitrectomy for epiretinal membrane and internal limiting membrane peeling. Medicine 2017; 96: e6709.

37. Lim HB, Lee MW, Kwak BS, et al. Longitudinal changes in thicknesses of the macula, ganglion cell-inner plexiform layer, and retinal nerve fiber layer after vitrectomy: a 12-month observational study. Retina 2017; 38: 155-162.

38. Wolf S, Schnurbusch U, Wiedemann P, et al. Peeling of the basal membrane in the human retina: ultrastructural effects. Ophthalmology 2004; 111: 238-243.

39. Pichi F, Lembo A, Morara M, et al. Early and late inner retinal changes after inner limiting membrane peeling. Int Ophthalmol 2014; 34: 437-446. 